\title{
Tissue-type plasminogen activator induces opening of the blood-brain barrier via the LDL receptor-related protein
}

\author{
Manuel Yepes,${ }^{1,2}$ Maria Sandkvist,${ }^{3}$ Elizabeth G. Moore, ${ }^{1}$ Thomas H. Bugge, ${ }^{4}$ \\ Dudley K. Strickland, ${ }^{1}$ and Daniel A. Lawrence ${ }^{1}$
}

${ }^{1}$ Department of Vascular Biology, Holland Laboratory, American Red Cross, Rockville, Maryland, USA
2Department of Neurology, Georgetown University Hospital, Washington, DC, USA
${ }^{3}$ Department of Biochemistry, Holland Laboratory, American Red Cross, Rockville, Maryland, USA
${ }^{4}$ Proteases and Tissue Remodeling Unit, Oral and Pharyngeal Cancer Branch, National Institute of Dental and Craniofacial
Research, NIH, Bethesda, Maryland, USA

The regulation of cerebrovascular permeability is critical for normal brain homeostasis, and the "breakdown" of the blood-brain barrier (BBB) is associated with the development of vasogenic edema and intracranial hypertension in a number of neurological disorders. In this study we demonstrate that an increase in endogenous tissue-type plasminogen activator (tPA) activity in the perivascular tissue following cerebral ischemia induces opening of the BBB via a mechanism that is independent of both plasminogen (Plg) and MMP-9. We also show that injection of tPA into the cerebrospinal fluid in the absence of ischemia results in a rapid dose-dependent increase in vascular permeability. This activity is not seen with urokinase-type Plg activator (uPA) but is induced in $\mathrm{Plg}^{-/-}$mice, confirming that the effect is Plgindependent. However, the activity is blocked by antibodies to the LDL receptor-related protein (LRP) and by the LRP antagonist, receptor-associated protein (RAP), suggesting a receptor-mediated process. Together these studies demonstrate that tPA is both necessary and sufficient to directly increase vascular permeability in the early stages of BBB opening, and suggest that this occurs through a receptor-mediated cell signaling event and not through generalized degradation of the vascular basement membrane. J. Clin. Invest. 112:1533-1540 (2003). doi:10.1172/JCI200319212.

\section{Introduction}

Outside the CNS, tissue-type plasminogen activator (tPA) is predominantly found in the blood, where its primary function is as a thrombolytic enzyme and its principal substrate is the zymogen plasminogen (Plg) (1). tPA is also expressed within the CNS, where it is thought to have a very different function, promoting events associated with synaptic plasticity such as motor learning, long-term potentiation, and seizures (2-5). The natural substrate for tPA within the CNS is controversial, with some studies indicating that its activity is Plg-dependent while others show it to be Plg-independent $(4,6)$.

Pathologically, tPA is linked to neurotoxicity in a number of settings $(6,7)$, but its mechanism of action is not known. The majority of studies suggest that

\section{Received for publication June 16, 2003, and accepted in revised form} September 30, 2003.

Address correspondence to: Daniel A. Lawrence, Vascular Biology Department, Holland Laboratory, American Red Cross, 15601 Crabbs Branch Way, Rockville, Maryland 20855, USA. Phone: (301) 517-0356; Fax: (301) 738-0794;

E-mail: Lawrenced@USA.redcross.org.

Conflict of interest: The authors have declared that no conflict of interest exists.

Nonstandard abbreviations used: tissue-type plasminogen activator (tPA); plasminogen (Plg); middle cerebral artery occlusion (MCAO); blood-brain barrier (BBB); LDL receptor-related protein (LRP); urokinase-type plasminogen activator (uPA); receptor-associated protein (RAP); N-methyl-Daspartate (NMDA); protease-activated receptor (PAR). excessive tPA within the CNS promotes neuronal death (8). However, at least one study has suggested that tPA is neuroprotective (9) and another has shown that tPA deficiency increases cerebrovascular fibrin deposition following transient middle cerebral artery occlusion (MCAO), and that this is associated with increased brain injury (10). In the case of ischemic stroke, endogenous tPA activity has been shown to increase significantly within the ischemic hemisphere following MCAO (8), and different animal models have demonstrated that both genetic deficiency of tPA $(8,11)$ and its inhibition with the natural tPA inhibitor neuroserpin $(7,12)$ are associated with significant neuronal survival and decreases in infarct volume compared with control animals.

These studies suggest that tPA inhibition within the CNS may be beneficial after ischemic stroke. At present, tPA is the only FDA-approved thrombolytic medication for the treatment of patients with acute ischemic stroke (13). However, in some animal models of embolic stroke, thrombolytic treatment with tPA was associated with evidence of increased vascular permeability $(14,15)$ and with increased infarct volume (14). This suggests that under pathological conditions there may be a correlation between tPA activity and changes in vascular permeability, and that any increases in vascular permeability could result in vascular tPA crossing into the CNS where it may intensify the effect of ischemia on excitotoxic cell death. 
The blood-brain barrier (BBB) is an incompletely understood structure that protects the brain from the entrance of potentially harmful substances present in the blood and maintains the homeostasis of the CNS (16). Regulation of BBB permeability is a necessary part of normal physiology; however, in pathologic situations such as head trauma and acute stroke, excessive increases in vascular permeability lead to opening of the BBB and vasogenic edema $(17,18)$. Previous studies have suggested that MMPs can promote this "breakdown" of the BBB by directly degrading the components of the vascular basement membrane (19). MMPs are zinc-dependent enzymes that are upregulated following ischemia (20). In particular, MMP-9 has been suggested to play a role in the pathology of stroke, and MMP-9-deficient mice ( $M M \mathrm{MP}^{-/-}$mice) have been shown to be partially protected from cerebral ischemia (21), purportedly due to reduced proteolytic degradation of the BBB (22).

The LDL receptor gene family is a group of related endocytic receptors (23). Members of this family are present on most cells and bind a diverse set of ligands, including tPA (24) and MMP-9 (25). The LDL receptor family has also been shown to participate in transendothelial transport through the $\operatorname{BBB}(26,27)$. Several members of this receptor family are highly expressed within the CNS, including the LDL receptor-related protein (LRP) (28). LRP and other members of this family have also been shown to participate in cell signaling events both in the vascular wall and in the CNS (28-30). In the studies presented here, we demonstrate that tPA within the CNS is both necessary and sufficient to directly increase vascular permeability, leading to loss of BBB integrity. Moreover, in initial stages of cerebral ischemia, the opening of the $\mathrm{BBB}$ is mediated directly by tPA; this activity is independent of either Plg or MMP-9, but requires interaction with LRP. These results describe a new role for $\mathrm{tPA}$ as a regulator of vascular permeability within the CNS.

\section{Methods}

Animal models. The middle cerebral artery was exposed in adult Sprague-Dawley rats weighing 350-400 g and was cauterized with a bipolar coagulator as described elsewhere (7). In mice, the middle cerebral artery was exposed and occluded with a 10-0 suture as described (11). Murine strains were $t P A^{-/-}$, urokinase-type Plg activator (uPA) null $\left(u \mathrm{PA}^{-/-}\right)$, or $\mathrm{Plg}^{-/-}$backcrossed at least seven generations into C57BL/6J mice (31); their WT C57BL/6J controls; MMP9-/- mice (32) on a pure 129S6/SvEv background (a generous gift from Michael Shipley and Robert Senior of Washington University School of Medicine, St. Louis, Missouri, USA); and their WT 129S6/SvEv controls (33) (Taconic Farms, Rockville, Maryland, USA). In the case of the $\mathrm{Plg}^{-/}$mice, all experiments were carried out on mice between 8 and 12 weeks of age. Treatment of rats with neuroserpin following MCAO was performed immediately after MCAO by intracortical injection with $20 \mu$ l of either PBS or 16 $\mu \mathrm{M}$ neuroserpin as described (7). Treatment of mice with neuroserpin following $\mathrm{MCAO}$ was performed by intraventricular injection of $2.5 \mu \mathrm{l}$ of neuroserpin (1-16 $\mu \mathrm{M})$ at bregma -2 , mediolateral 0 , and dorsoventral 2 (34). All animal experiments were approved by the Institutional Animal Care and Use Committee at the American Red Cross Holland Laboratory.

MMP-9 activity analysis. For analysis of MMP-9 activity in rats and mice, animals were euthanized 6 hours after MCAO. The brains were then removed, divided into ipsilateral and contralateral hemispheres, and homogenized in a 2:1 volume-to-weight ratio of lysis buffer containing $0.5 \mathrm{M} \mathrm{NaCl}, 1 \%$ Triton X-100 (Sigma-Aldrich, St. Louis, Missouri, USA), $0.05 \%$ Tween 20 (Sigma-Aldrich), and 50 $\mathrm{mM}$ sodium acetate at $\mathrm{pH} 4$ (7). The protein concentrations of the extracts were determined and the samples were diluted to identical concentrations in PBS before MMP activity was assayed by gelatin zymography (Invitrogen Corp., Carlsbad, California, USA). After electrophoresis, the gel was washed twice (45 minutes each wash) with $2.5 \%$ Triton X-100 (Sigma-Aldrich), followed by a 30 -minute wash with Tris-buffered saline containing $5 \mathrm{mM} \mathrm{CaCl}_{2}$. The gel was then incubated overnight at $37^{\circ} \mathrm{C}$ in the same buffer, after which it was stained with 0.5\% Coomassie Brilliant Blue R-250 (Amersham Biosciences, Piscataway, New Jersey, USA) for 30 minutes and then destained. Purified human proMMP-9 from Chemicon International (Temecula, California, USA) was used as a marker for MMP-9. Treatment of brain extracts from animals subjected to MCAO with para-aminophenyl mercuric acetate (Sigma-Aldrich) did not increase the amount of MMP-9 activity or alter its migration in the zymography assays, suggesting that the majority of MMP-9 observed was in the active form (data not shown).

Analysis of vascular permeability. Changes in vascular permeability following MCAO in mice were determined by intravenous injection of 2\% Evans blue (SigmaAldrich) in saline immediately after MCAO, followed 6 hours later by transcardiac perfusion. The brains were then removed, divided into ipsilateral and contralateral hemispheres, weighed, homogenized in $400 \mu \mathrm{l}$ of $N, N$ dimethylformamide (Sigma-Aldrich), and centrifuged at $21,000 \mathrm{~g}$ for 30 minutes. Evans blue was quantified from the absorbance at $620 \mathrm{~nm}$ of each supernatant minus the background calculated from the baseline absorbance between 500 and $740 \mathrm{~nm}$, and divided by the wet weight of each hemisphere. To determine vascular permeability following intraventricular injection of tPA or uPA, anesthetized mice, either WT or $\mathrm{Plg}^{-1-}$ C57BL/6J, were placed on a stereotactic frame and injected at coordinates bregma -2 , mediolateral 0 , and dorsoventral 2 (34). Injections contained $2.5 \mu \mathrm{l}$ of either active tPA (0.14-3.4 $\mu \mathrm{M}$; Genentech Inc., San Francisco, California, USA; $)$, inactive tPA $(0.7 \mu \mathrm{M}$; Molecular Innovations Inc., Royal Oak, Michigan, USA), or uPA (0.9 $\mu \mathrm{M}$; Molecular Innovations Inc.) and were followed by the intravenous injection of $2 \%$ Evans blue.

The role of LRP in vascular permeability was analyzed in WT C57BL/6J mice injected at the same coordinates with $5 \mu \mathrm{l}$ of a combination of active tPA $(0.35 \mu \mathrm{M})$ and 
either receptor-associated protein (RAP) $(9 \mu \mathrm{M})$, purified goat anti-LRP antibodies $(85 \mu \mathrm{g} / \mathrm{ml})$, or normal goat IgG $(85 \mu \mathrm{g} / \mathrm{ml})$. The role of the $\mathrm{N}$-methyl-D-aspartate (NMDA) receptor was analyzed in a different group of animals, which were first injected intraperitoneally with $100 \mu \mathrm{l}$ of the NMDA antagonist MK-801 $(2 \mathrm{mg} / \mathrm{ml}$; Tocris Cookson Inc., Ellisville, Missouri, USA) 1 hour before intraventricular injection of $\mathrm{tPA}(2.5 \mu \mathrm{l}$ of $0.7 \mu \mathrm{M}$ active $\mathrm{tPA}$ ). The inactive $\mathrm{PPA}$ (with its active site blocked) was prepared by inhibition of tPA with Phe-Pro-Argchloromethylketone (EMD Biosciences Inc., La Jolla, California, USA), followed by dialysis into the same buffer as the active tPA. For all groups, brains were extracted 1 hour after intraventricular injection and Evans blue extravasation was analyzed as above, except that brains were not separated into hemispheres and were extracted in $800 \mu \mathrm{l}, N$-dimethylformamide. Statistical analyses for all quantitative experiments were performed with the Wilcoxon two-sample rank sum test. Microscopy and in situ proteinase analysis. Microscopic evaluation of in situ proteinase activity and Evans blue dye extravasation were determined at 1 hour or 6 hours after MCAO, respectively. In both cases mice were intracardially perfused with PBS, followed by a brief perfusion with $10 \%$ paraformaldehyde (Fisher Scientific Co., Fairlawn, New Jersey, USA) in PBS and then again extensively with PBS alone. The brains were then removed, frozen in OCT (Sigma-Aldrich, St. Louis, Missouri, USA), and stored at $-70^{\circ} \mathrm{C}$. For analysis of tPA, $5-\mu \mathrm{m}$ cryostat sections were analyzed as described (4). Briefly, cryostat sections $(5 \mu \mathrm{m})$ were first stained with $100 \mu \mathrm{M}$ DAPI (Sigma-Aldrich) for 30 seconds, rinsed in PBS, and then processed for in situ proteinase activity. Overlays of 100 $\mu \mathrm{l}$ of $1 \%$ agarose in PBS containing $1 \mu \mathrm{g} / \mathrm{ml}$ BODIPY TR$\mathrm{X}$ casein (Molecular Probes Inc., Eugene, Oregon, USA) and $5 \mathrm{mM}$ EDTA with or without Plg (Molecular Innovations Inc.) and with or without anti-tPA antibodies were applied to prewarmed tissue and sealed under glass coverslips. The slides were incubated for 2 hours at $37^{\circ} \mathrm{C}$ and then analyzed for casein fluorescence. To study Evans blue extravasation, $5-\mu \mathrm{m}$ brain sections were processed in the same manner except that the overlay contained only $1 \%$ agarose and was analyzed immediately after preparation. Evans blue was observed using a fluorescent microscope with a TRITC filter.

\section{Results}

Inbibition of $t P A$ reduces ischemia-induced cerebral MMP-9 activity. Previous studies have shown that the activity of MMP-9 increases in the CNS following cerebral ischemia $(20,21)$ and that compared with WT mice, $M M P 9^{-/-}$mice have both reduced infarct volumes and decreased disruption of the BBB after MCAO (35). Like MMP-9, tPA activity in the CNS also increases early after MCAO (7), and $t P A^{-/-}$mice show reductions in stroke volume similar to $M M P 9^{-/-}$mice (8). The similarity of results obtained with these two protease systems suggests that there may be a link between tPA and MMP-9 during cerebral ischemia. To test this possibility, experiments were conducted to see if the inhibition of tPA activity within the CNS after MCAO influenced the levels of MMP-9 present. For this analysis, gelatin zymographic assays of rat brains were performed 6 hours after MCAO with and without injection of the tPA inhibitor neuroserpin directly into the ischemic area (Figure 1a). These results revealed a marked decrease in MMP-9 activity in neuroserpin-treated animals (lanes 4-7) compared with animals treated with PBS only (lane 2 and lane 3 ). Treatment of brain extracts with neuroserpin ex vivo indicated that, as expected, neuroserpin did not directly inhibit MMP-9 (data not shown), which suggests that the inhibition of tPA activity indirectly blocks the rise in MMP-9 activity that occurs after ischemic stroke. To confirm that this result was due to the inhibition of tPA activity in the neuroserpin-treated animals and was not due to the effect of neuroserpin on another unidentified protease, the MMP-9 activity was analyzed in $\mathrm{PPA}^{-/-}$and $\mathrm{Plg}^{-/-}$ mice 6 hours after MCAO. These data demonstrated that compared with WT animals, $t P A^{-/-}$mice showed a marked decrease in MMP-9 activity, whereas $\mathrm{Plg}^{-/}$mice did not (Figure 1b). Quantitative PCR analysis of brain tissue from normal WT mice and from the ischemic and nonischemic hemispheres of WT mice 6 hours after MCAO indicated that there was no local increase in MMP9 gene expression following cerebral ischemia (data not shown). Likewise, treatment of either nonischemic brain extracts or purified human proMMP-9 with tPA demonstrated that tPA had no affect on the MMP-9 in either case, indicating that $\mathrm{tPA}$ was not acting directly on MMP-9 (data not shown). Together these results suggest

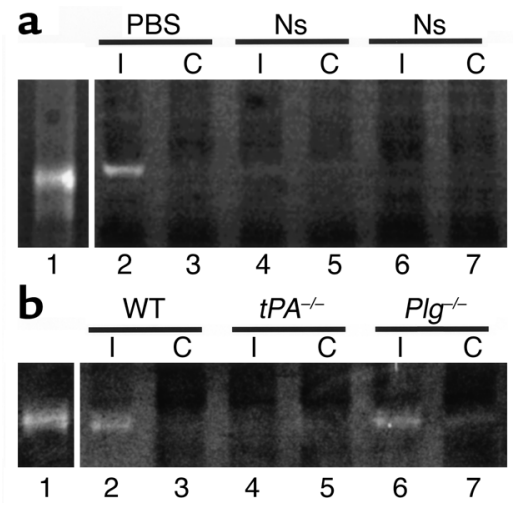

Figure 1

MMP-9 activity in brain extracts following cerebral ischemia. (a) Zymographic assay of brain extracts from rats following MCAO. Lane 1 is purified human proMMP-9 and all other lanes show MMP- 9 activity 6 hours after MCAO in rats that were intracortically injected ipsilateral to the ischemic area immediately following MCAO with either PBS (lanes 2 and 3 ) or neuroserpin (Ns, lanes 4-7). Lanes 2, 4, and 6 are ipsilateral (I) to the ischemic area and lanes 3, 5, and 7 are contralateral (C). (b) Zymographic assay of brain extracts from mice following MCAO. Lane 1 is purified human proMMP-9. The other lanes correspond to brain extracts from WT C57BL/6J (lane 2 and lane 3), $t P A^{-/-}$C57BL/6J (lane 4 and lane 5), and $P / g^{-1-} \mathrm{C} 57 \mathrm{BL} / 6 \mathrm{~J}$ (lane 6 and lane 7) mice 6 hours after MCAO. Lanes 2, 4, and 6 are ipsilateral to the ischemic area and lanes 3, 5, and 7 are contralateral. 


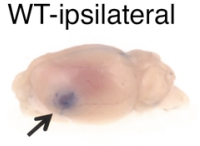

$u P A^{--}$

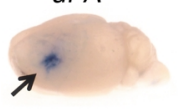

b

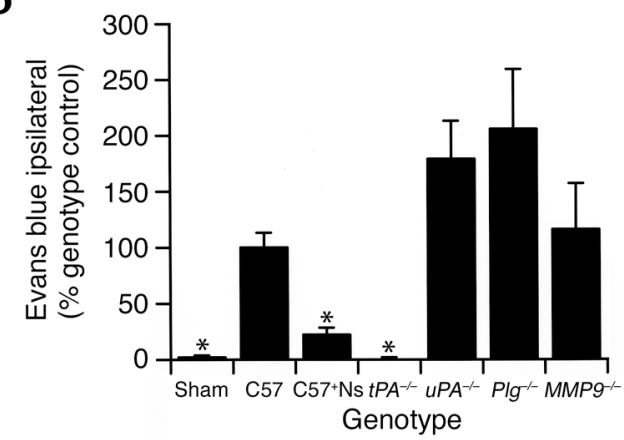

Figure 2

BBB permeability following cerebral ischemia. (a) Analysis of Evans blue dye extravasation in mice. Brains were removed and photographed 6 hours after MCAO. All images are ipsilateral to the ischemic area. WT ipsilateral is a WT C57BL/6J mouse, and WT + Ns is a WT C57BL/6 mouse treated with an intraventricular injection of $2.5 \mu \mathrm{l}$ of $16 \mu \mathrm{M}$ neuroserpin immediately after MCAO. $t P A^{-/-}, u P A^{-/-}$, $\mathrm{Plg}^{-1-}$, and $\mathrm{MMP9}^{-/-}$are mice deficient in the enzyme indicated. The arrows indicate the area of Evans blue extravasation associated with the ischemic injury. (b) Quantitative analysis of Evans blue extravasation from brain extracts 6 hours after MCAO. Sham indicates animals subjected to all procedures except MCA ligation. The results represent the specific absorbance of Evans blue at $620 \mathrm{~nm}$ calculated as percentage of that of the WT control, either C57BL/6) (C57) or 129S6/ SvEv as described in Methods. As a control for perfusion efficiency, the absorbance of the contralateral hemisphere was subtracted from that of the hemisphere ipsilateral to the MCAO. For each condition, $n=4$. ${ }^{*} P<0.05$ relative to $\mathrm{WT}$ control mice receiving MCAO.

that there is a Plg-independent link between tPA and MMP-9 following cerebral ischemia that is not due to the local action of tPA on either MMP-9 protein or gene expression. Instead it may be that the rise in MMP- 9 was due to the increased passage of free MMP-9 from the blood into the brain through the BBB or as a component of invasive neutrophils.

tPA increases BBB permeability after MCAO independent of $M M P-9$. If the rise in MMP-9 activity observed above is due to the passage of MMP-9 from the blood through the $\mathrm{BBB}$, this suggests that $\mathrm{PAA}$ may play an important role in the regulation of vascular permeability following MCAO. To test this hypothesis we analyzed Evans blue dye extravasation in WT, $\mathrm{PA}^{-/-}, \mathrm{Plg}^{-/-}, u \mathrm{PA}^{-/-}$, and $M M P 9^{-/-}$mice 6 hours after MCAO (Figure 2, a and b). These studies demonstrated that there was a large increase in BBB permeability in the ischemic area in WT animals by 6 hours after MCAO that was not seen in sham-operated mice. This increased permeability was dramatically reduced in both $t P A^{-/-}$mice and in WT mice treated with neuroserpin, suggesting a direct association between $\mathrm{PAA}$ and $\mathrm{BBB}$ opening in cerebral ischemia.
In contrast, ischemia-induced Evans blue dye leakage was not reduced in either $u \mathrm{PA}^{-/-}$or $\mathrm{Plg}^{-/-}$animals, indicating that $\mathrm{tPA}$ induces opening of the BBB by a Plg-independent mechanism. Moreover, MMP-9-deficient animals also exhibited Evans blue dye extravasation comparable to that of WT animals, demonstrating that at least in the early stages of cerebral ischemia the opening of the BBB is mediated by tPA and is independent of MMP-9. To confirm that the effect of neuroserpin on Evans blue dye leakage was specific and was not simply due to the injection or was an effect of the vehicle, a doseresponse study of neuroserpin was performed. These data are shown in Figure 3 and indicate that Evans blue dye leakage decreases as the concentration of neuroserpin increases, and saturates at a point where higher concentrations of neuroserpin do not have any additional effect.

Perivascular $t P A$ activity precedes changes in $B B B$ integrity following $M C A O$. To determine whether the observed effect of tPA on the BBB following cerebral ischemia might be due to the local action of tPA directly on the blood vessels, the temporal and spatial relationship between tPA activity and Evans blue dye leakage following MCAO was examined by in situ zymography and fluorescence microscopy. These studies demonstrated that as early as 1 hour after MCAO, there was a robust increase in tPA activity associated with the vessel and in the perivascular tissue surrounding the necrotic core (ischemic penumbra), as evidenced by the generation of plasmin activity in the in situ zymography assay (Figure 4a). This activity preceded an increase in vascular permeability in the same area shown 5 hours later in Figure 4f (6 hours after MCAO). These results suggest a direct temporal and spatial relationship between tPA activity and increased permeability of the $\mathrm{BBB}$ following the onset of cerebral ischemia.

$t P A$ directly induces opening of the $B B B$. The studies described above indicate that tPA is necessary to induce opening of the BBB following cerebral ischemia. To determine whether tPA by itself is sufficient to induce opening of the BBB in the absence of other stimuli such

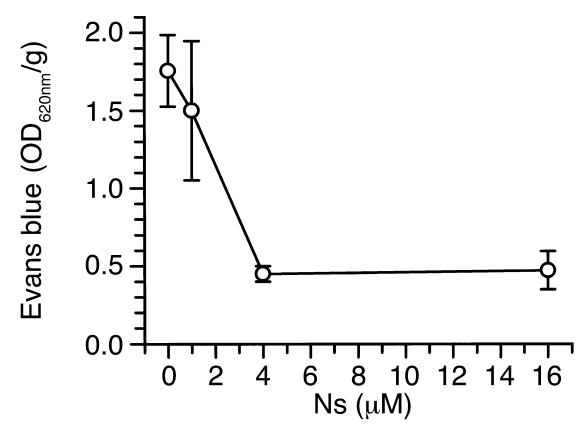

\section{Figure 3}

Dose-response study of neuroserpin treatment following MCAO. Quantitative analysis of Evans blue extravasation from brain extracts 6 hours after MCAO in WT C57BL/6J mice treated with an intraventricular injection of $2.5 \mu \mathrm{l}$ of either PBS or increasing doses of neuroserpin immediately after MCAO. Analysis was as in Figure 2, and $n=4-6$ for each point. 

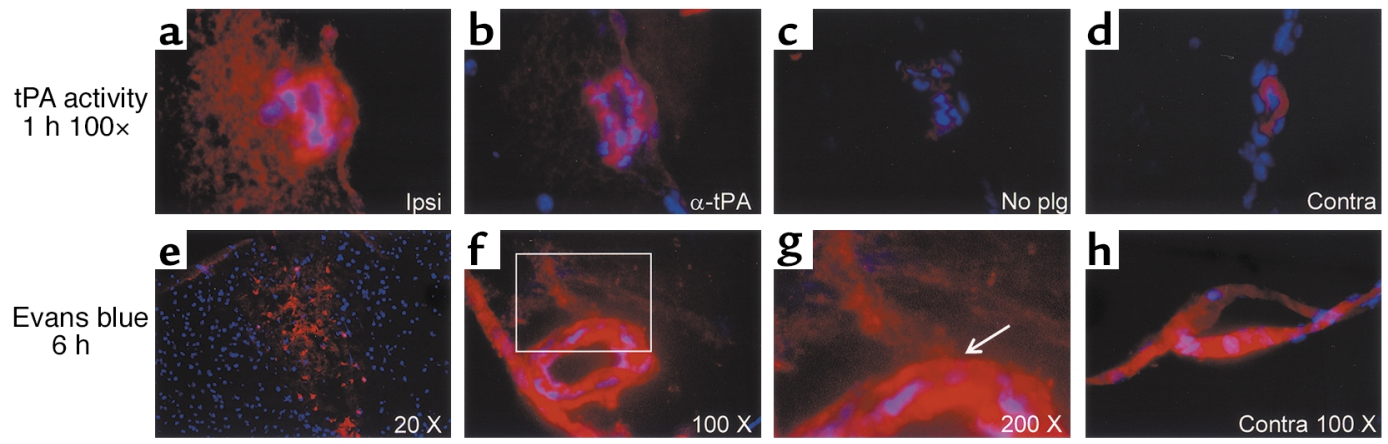

\section{Figure 4}

Temporal and spatial relationship between tPA activity and vascular permeability following MCAO in WT (C57BL/6J) mice. In a-d, tPA activity 1 hour after MCAO is shown in red by in situ zymography, and cell nuclei are in blue (DAPI). (a) By 1 hour after MCAO there is significant tPA activity in the vessel wall and in the perivascular tissue surrounding a vessel bordering the necrotic area. (b and c) The same vessel in adjacent sections $(5 \mu \mathrm{m})$, but with anti-tPA antibodies included (b), or without the addition of Plg (c) in the overlay. (d) The background tPA activity associated with a vessel in a corresponding area in the contralateral hemisphere from the same section shown in a. In a-d the original magnification was $\times 100$. (e-h) Evans blue extravasation is shown in red and cell nuclei in blue (DAPI) 6 hours after MCAO. (e) A low-magnification view of the entire ischemic area. (f) Evans blue extravasation from a vessel located in the area adjacent to the ischemic area, similar to the one seen in $\mathbf{a}$. (g) Electronic magnification of the box in $\mathbf{f}$. The arrow indicates an area of Evans blue leakage outside the internal elastic lamina of the vessel. (h) Evans blue is shown adhering to the vessel wall, but no extravasation is seen in a vessel from the same section seen in $\mathbf{f}$ and $\mathbf{g}$ but located in the corresponding region of the contralateral hemisphere. Ipsi, ipsilateral; Contra, contralateral.

as ischemia, tPA was injected directly into the CSF, and Evans blue dye extravasation was studied 1 hour later. This analysis showed that there was a significant increase in vascular permeability following the intraventricular injection of tPA in the absence of cerebral ischemia (Figure 5). In addition, this effect was dosedependent, with an estimated $\mathrm{ED}_{50}$ of approximately $425 \mathrm{nM}$ tPA (Figure 6). These results indicate that tPA within the CNS is not only necessary, but is sufficient to produce opening of the BBB. Moreover, very little leakage of Evans blue dye was observed in the presence of inactive tPA, indicating that the tPA must be proteolytically active to induce opening of the BBB (Figure 5). However, in contrast to active $\mathrm{PA}$, the injection of active uPA induced only a modest increase in Evans blue extravasation, suggesting that like the tPA-dependent increases in vascular permeability associated with stroke, this event may also be Plg-independent. To test this latter possibility, tPA was injected directly into the $\mathrm{CSF}$ of $\mathrm{Plg}^{-/}$mice, and Evans blue dye extravasation was studied after 1 hour. These data demonstrated that just as in WT mice, tPA significantly increased the vascular permeability in the absence of Plg (white bar in Figure 5), confirming that this effect is Plg-independent.

tPA-induced opening of the BBB is blocked by antagonists of LRP. tPA has been reported to associate with two non$\mathrm{Plg}$ ligands within the CNS and to mediate cell signaling through these interactions. These receptors are the NR-1 subunit of the NMDA receptor (36), although this association remains controversial (37), and an LDL receptor family member presumed to be LRP (24). To determine whether the observed increase in $\mathrm{BBB}$ permeability was due to the association of tPA with either the NR-1 subunit of the NMDA receptor or with LRP, the leakage of Evans blue dye following the intraventricular injection of tPA was evaluated with antagonists to both receptor families. In the first case, mice were pretreated 1 hour before tPA administration with the NMDA receptor antagonist MK-801 (38). These experiments indicated that there was no significant change in the tPAinduced increase in BBB permeability in the MK-801treated animals (Figure 5), suggesting that the observed increase in BBB permeability following treatment with tPA is not mediated by a member of the NMDA receptor family. In contrast, intraventricular coinjection of tPA together with RAP, a general antagonist of members of

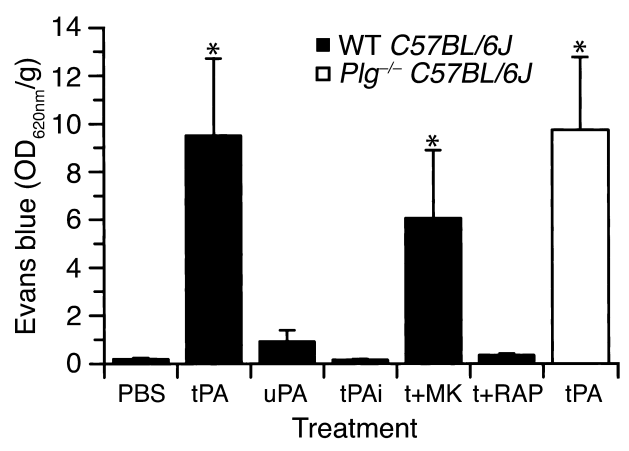

Figure 5

Changes in vascular permeability following intraventricular injection of tPA, determined by quantitative analysis of Evans blue extravasation from brain extracts. Measurements were performed 1 hour after intraventricular injection of $2.5 \mu \mathrm{l}$ of either PBS, active tPA (tPA, 0.7 $\mu \mathrm{M})$, inactive tPA ( $\mathrm{tPAi}, 0.7 \mu \mathrm{M})$, active $\mathrm{UPA}$ ( $\mathrm{uPA}, 0.9 \mu \mathrm{M})$, and active $\mathrm{tPA}$ following 1 hour after pretreatment with the NMDA receptor antagonist MK-801 ( $\mathrm{t}+\mathrm{MK})$ or coinjection of $5 \mu \mathrm{l}$ of $0.35 \mu \mathrm{M}$ active $\mathrm{TPA}$ in combination with a 25 -fold molar excess $(9 \mu \mathrm{M})$ of the LRP antagonist RAP ( $t+R A P)$. The black bars indicate WT C57BL/6J mice and the white bar indicates $\mathrm{Plg} /-\mathrm{C} 57 \mathrm{BL} / 6 \mathrm{~J}$ mice. For each condition, $n=6-8 .{ }^{*} P<0.01$ vs. animals injected with PBS. 


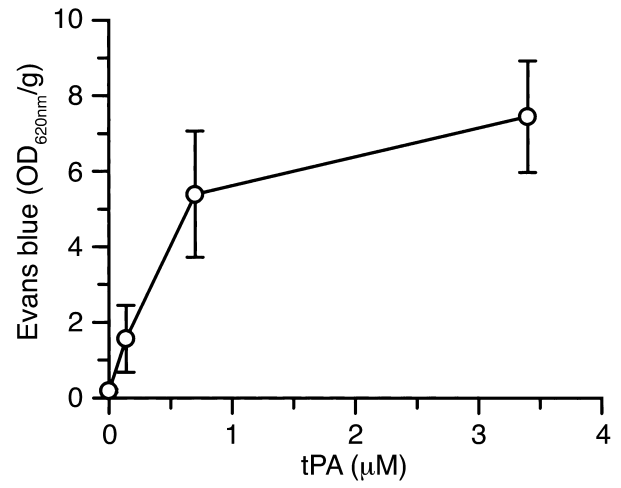

\section{Figure 6}

Analysis of Evans blue extravasation following a dose-response study of intraventricular injection of tPA. Quantitative analysis of Evans blue extravasation from brain extracts of WT C57BL/6J mice 1 hour after intraventricular injection of $2.5 \mu \mathrm{l}$ of increasing concentrations of active tPA. $n=6$ for each point.

the LDL receptor family (39), completely blocked the extravasation of Evans blue dye (Figure 5). This indicates that the action of tPA on the BBB was mediated by LRP or by another member of the LDL receptor family. To determine whether $\mathrm{TPA}$ was acting specifically through LRP, Evans blue extravasation was determined when tPA was coadministered with anti-LRP antibodies (Figure 7). These results demonstrated that antibodies against LRP completely blocked this effect of tPA, confirming the role of LRP in this process.

\section{Discussion}

The BBB is formed by endothelial cells, pericytes, astrocytes, and the vascular basement membrane. Its main functions are to form a tight barrier that regulates the entry of selected molecules from the blood into the CNS and to prevent the passage of potentially harmful substances into the brain $(40,41)$. Changes in BBB permeability are a normal physiological response to events such as variations in $\mathrm{pH}$, blood pressure, and the supply of oxygen and nutrients. However, an excessive and sustained increase in vascular permeability may produce an abnormal and enduring opening of the BBB, leading to the extravasation and accumulation of fluids in the perivascular space, resulting in vasogenic edema $(17,18)$.

The causes of the pathologic opening of the BBB are not known, but previous studies have suggested that in the case of cerebral ischemia it is the upregulation of MMP-9 and its subsequent proteolytic degradation of the vascular basement membrane that leads to breakdown of the BBB (22). Our initial observations of a progressive increase in MMP-9 activity within the ischemic hemisphere after MCAO were consistent with this hypothesis. However, the absence in our experiments of a local increase in $M M P 9$ gene expression following permanent MCAO without reperfusion suggests that the observed increase in MMP-9 activity is more likely due to the passage of MMP-9 from the blood into the brain through an already compromised BBB. The results obtained with the $M M P^{-1-}$ mice, which exhibited an early extravasation of Evans blue dye similar to that observed in WT animals, are consistent with this interpretation and demonstrate that, at least in the very early stages of BBB opening following MCAO, MMP-9 does not appear to play a significant role. Nevertheless, these results clearly do not rule out a role for MMP-9 in cerebral ischemia, and MMP-9 may play an important part in the advanced stages of stroke-induced breakdown of the $\operatorname{BBB}(21,22)$. Still, our data do indicate that the early increase in tPA activity associated with the vessel and in the perivascular tissue is necessary for the initial opening of the BBB and that this may precede the later passage of MMP-9 into the CNS. Two observations seem to support this hypothesis. First, thrombolytic treatment with tPA in rats following embolic stroke is associated with a greater increase in MMP-9 activity compared with animals undergoing mechanical reperfusion (15), and second, increased MMP-9 activity is a marker of hemorrhagic complications after thrombolysis with tPA (42).

In contrast to the results obtained with the $M M P 9^{-/-}$ mice, the early increase in vascular permeability following cerebral ischemia was significantly reduced in $t P A^{-/-}$mice and in animals treated with neuroserpin. These results demonstrate that in the first stages of cerebral ischemia, tPA is required for the initial opening of the BBB. It has been previously shown that within 6 hours after the onset of cerebral ischemia there is a local increase in endogenous tPA activity in the ischemic area (7). In the current study we extended this result and demonstrated that this rise occurred as early as 1 hour after MCAO, and that it first occurred precisely in the perivascular tissue within the ischemic penumbra where the extravasation of Evans blue dye was observed 5 hours later.

Since an increase in vascular permeability is very likely the first step in the opening of the BBB, it is possible to hypothesize a direct role for tPA as a natural regulator of vascular tone and permeability. In pathological

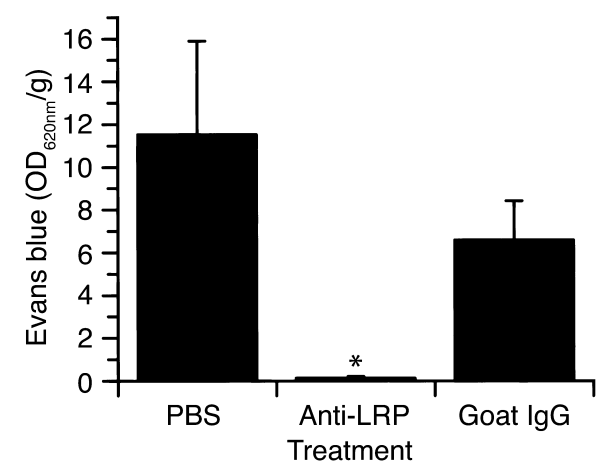

Figure 7

Effect of anti-LRP antibodies on tPA-induced cerebrovascular permeability determined by quantitative analysis of Evans blue extravasation from brain extracts of WT C57BL/6J mice 1 hour after intraventricular coinjection of $5 \mu \mathrm{l}$ of $0.35 \mu \mathrm{M}$ tPA with either PBS, purified goat anti-LRP IgG $(85 \mu \mathrm{g} / \mathrm{ml})$, or normal goat IgG $(85$ $\mu \mathrm{g} / \mathrm{ml}) . n=6$ for each point. ${ }^{*} P<0.01$ vs. animals injected with PBS. 
conditions such as stroke, the ischemic insult may induce the release of tPA from activated microglial cells (and possibly other cell types), and this tPA may act as a signal to the perivascular smooth muscle cells or other vascular cells to increase vascular permeability. However, if the ischemia is not relieved and the signal continues, then its persistence and amplification could lead to BBB opening and vasogenic edema. This hypothesis is supported by the reported association between thrombolytic treatment with tPA and radiological (14) and pathological (15) evidence of BBB opening in embolic stroke.

The demonstration that the injection of tPA directly into the CSF, in the absence of cerebral ischemia, is sufficient to cause a significant increase in vascular permeability strongly supports the hypothesis that tPA may be a natural regulator of vascular tone and permeability. The mechanism of this action is not clear. However, the observations that no leakage of Evans blue dye was observed in the presence of inactive tPA and that the leakage following $\mathrm{MCAO}$ was blocked by treatment with the tPA inhibitor neuroserpin indicates that tPA's proteolytic activity is required. These results also indicate that tPA within the CNS is both necessary and sufficient to produce opening of the BBB. Moreover, this effect is independent of Plg since no differences in the tPA-induced changes in vascular permeability were observed between WT and $\mathrm{Plg}^{-1-}$ mice. We hypothesize that tPA injected into the CSF, perhaps through a receptor-mediated mechanism, interacts with cells in the subependymal layer and either transduces a signal directly to the vessel or is transported into the perivascular tissue where it interacts with either the pericytes or the subvascular astrocytes/microglia. This interaction then induces the increase in vascular permeability, most likely through a receptor-mediated signaling event. Previous reports have suggested that tPA can interact with two different receptors known to be present within the CNS, the NR-1 subunit of the NMDA receptor (36) and LRP (24). Our studies indicated that there was no significant decrease in the tPA-induced increase in BBB permeability in animals treated with an NMDA receptor antagonist, but that the LDL receptor family antagonist RAP and specific anti-LRP antibodies completely blocked tPA's effects. This suggests that tPA does not act through the NMDA receptor and instead its action is mediated by LRP.

Taken together, these data suggest a model for tPA as a regulator of vascular permeability that requires tPA's proteolytic activity but is both Plg-and MMP-9-independent. Furthermore, these results describe a unique role for tPA within the CNS that is not observed with $\mathrm{uPA}$, and suggests that the substrate must either be very specific for $\mathrm{tPA}$ or that the substrate is largely inaccessible to uPA. In the case of cerebral ischemia, it appears as though an early rise in tPA activity in the subvascular tissue, possibly released from activated microglial cells (43), results in the association of this tPA with LRP, possibly located on the perivascular smooth muscle cells or pericytes.
Interestingly, previous studies have suggested that LRP can mediate intracellular signaling both in the CNS, where it modulates long-term potentiation (29), and in vascular smooth muscle cells, where it regulates cell proliferation and protects vascular wall integrity through control of PDGF receptor activation (30). Thus, it is likely that the association of tPA with LRP induces an increase in vascular permeability via a specific cell signaling event, and if the signal persists or is too robust, then this increased permeability leads to opening of the BBB and vasogenic edema.

The importance of tPA's proteolytic activity in the signaling process, in addition to its LRP binding activity, is indicated by two observations. First, that chemically inactivated tPA does not promote increased vascular permeability (Figure 5) even though it binds to LRP with similar affinity as active tPA (data not shown); and second, that neuroserpin can block the tPA-dependent Evans blue extravasation following $\mathrm{MCAO}$. This latter observation is important since neuroserpin is known to inhibit the protease activity of tPA but does not inhibit its binding to LRP (data not shown). Together these data imply a two-step process in which tPA binds to LRP on the cell surface, and when bound, cleaves an as-yet-unidentified substrate.

One possible candidate substrate is LRP itself, since recent studies have shown that the cytoplasmic domain of LRP can be released from the membrane through a process that involves proteolytic processing of the extracellular domain of LRP, and that the free intracellular domain can then regulate cell signaling pathways (44). Clearly, blocking either the proteolytic activity of tPA or its association with LRP could prevent this potential processing. Other possible substrates are members of the family of proteaseactivated receptors (PARs). These are G protein-coupled receptors that are activated by proteolytic cleavage of their extracellular amino terminus (45), and previous studies have shown that the activation of one member of this family, PAR-2, results in rapid vasodilation of cerebral arteries (46). We could speculate that a putative tPA cleavage site on a PAR is inaccessible to free tPA, but that when tPA is bound to LRP the cleavage is facilitated. Likewise, as discussed above for LRP, blocking either the proteolytic activity of tPA or its association with LRP would prevent this potential processing. The reason that uPA would not show activity toward either substrate is less clear; however, we note that it is not known whether uPA and tPA associate with the same site on LRP. Obviously, these are not the only possible substrates for tPA, but they do represent intriguing potential targets for further investigation of this process.

Finally, our data suggest that tPA may also promote vasogenic edema in other neurological pathologies such as subarachnoid hemorrhage, where the presence of blood-borne tPA in the subarachnoid space may promote increased vascular permeability with subsequent cerebral edema. Likewise, this process 
may also play a role in vascular permeability outside of the CNS, since tPA antigen has been observed in the sympathetic axons innervating the smooth muscle in the walls of peripheral vessels (47), which are also know to express significant levels of LRP (30, 48). Thus, our results imply a new Plg-independent role for tPA as a potent but previously unrecognized regulator of vascular permeability.

\section{Acknowledgments}

This work was supported by NIH grants HL-55374, HL-55747, and HL-54710 to D.A. Lawrence and NS02223 to M. Yepes.

1. Bugge, T.H., et al. 1996. Loss of fibrinogen rescues mice from the pleiotropic effects of plasminogen deficiency. Cell. 87:709-719.

2. Qian, Z., Gilbert, M.E., Colicos, M.A., Kandel, E.R., and Kuhl, D. 1993. Tissue-plasminogen activator is induced as an immediate-early gene during seizure, kindling and long-term potentiation. Nature. 361:453-457.

3. Seeds, N.W., Williams, B.L., and Bickford, P.C. 1995. Tissue plasminogen activator induction in Purkinje neurons after cerebellar motor learning. Science. 270:1992-1994.

4. Yepes, M., et al. 2002. Regulation of seizure spreading by neuroserpin and tissue-type plasminogen activator is plasminogen-independent. J. Clin. Invest. 109:1571-1578. doi:10.1172/JCI200214308.

5. Mataga, N., Nagai, N., and Hensch, T.K. 2002. Permissive proteolytic activity for visual cortical plasticity. Proc. Natl. Acad. Sci. U. S. A 99:7717-7721

6. Tsirka, S.E., Gualandris, A., Amaral, D.G., and Strickland, S. 1995. Excitotoxin-induced neuronal degeneration and seizure are mediated by tissue plasminogen activator. Nature. 377:340-344.

7. Yepes, M., et al. 2000. Neuroserpin reduces cerebral infarct volume and protects neurons from ischemia-induced apoptosis. Blood. 96:569-576.

8. Wang, Y.F., et al. 1998. Tissue plasminogen activator (tPA) increases neuronal damage after focal cerebral ischemia in wild-type and tPA-deficient mice. Nat. Med. 4:228-231.

9. Kim, Y.H., Park, J.H., Hong, S.H., and Koh, J.Y. 1999. Nonproteolytic neuroprotection by human recombinant tissue plasminogen activator. Science. 284:647-650.

10. Tabrizi, P., et al. 1999. Tissue plasminogen activator (tPA) deficiency exacerbates cerebrovascular fibrin deposition and brain injury in a murine stroke model: studies in tPA-deficient mice and wild-type mice on a matched genetic background. Arterioscler. Thromb. Vasc. Biol. 19:2801-2806

11. Nagai, N., De Mol, M., Lijnen, H.R., Carmeliet, P., and Collen, D. 1999. Role of plasminogen system components in focal cerebral ischemic infarction: a gene targeting and gene transfer study in mice. Circulation. 99:2440-2444.

12. Cinelli, P., et al. 2001. Neuroserpin, a neuroprotective factor in focal ischemic stroke. Mol. Cell. Neurosci. 18:443-457.

13. 1995. Tissue plasminogen activator for acute ischemic stroke. The National Institute of Neurological Disorders and Stroke rt-PA Stroke Study Group. N. Engl. J. Med. 333:1581-1587.

14. Zhang, Z., et al. 2002. Adjuvant treatment with neuroserpin increases the therapeutic window for tissue-type plasminogen activator administration in a rat model of embolic stroke. Circulation. 106:740-745.

15. Aoki, T., Sumii, T., Mori, T., Wang, X., and Lo, E.H. 2002. Blood-brain barrier disruption and matrix metalloproteinase- 9 expression during reperfusion injury: mechanical versus embolic focal ischemia in spontaneously hypertensive rats. Stroke. 33:2711-2717.

16. Rubin, L.L., and Staddon, J.M. 1999. The cell biology of the blood-brain barrier. Annu. Rev. Neurosci. 22:11-28.

17. Garcia, J.H., Lossinsky, A.S., Kauffman, F.C., and Conger, K.A. 1978 Neuronal ischemic injury: light microscopy, ultrastructure and biochemistry. Acta Neuropathol. (Berl.) 43:85-95.

18. Baker, R.N., Cancilla, P.A., Pollock, S., and Frommes, S.P. 1971. The movement of exogenous protein in experimental cerebral edema. An electron microscopic study after freeze-injury. J. Neuropathol. Exp. Neurol. 30:668-679.

19. Romanic, A.M., and Madri, J.A. 1994. Extracellular matrix-degrading proteinases in the nervous system. Brain Pathol. 4:145-156.

20. Rosenberg, G.A., Navratil, M., Barone, F., and Feuerstein, G. 1996. Proteolytic cascade enzymes increase in focal cerebral ischemia in rat. J. Cereb. Blood Flow Metab. 16:360-366.
21. Asahi, M., et al. 2000. Role for matrix metalloproteinase 9 after focal cerebral ischemia: effects of gene knockout and enzyme inhibition with BB-94. J. Cereb. Blood Flow Metab. 20:1681-1689.

22. Asahi, M., et al. 2001. Effects of matrix metalloproteinase-9 gene knockout on the proteolysis of blood-brain barrier and white matter components after cerebral ischemia. J. Neurosci. 21:7724-7732.

23. Herz, J., et al. 1988. Surface location and high affinity for calcium of a 500 -kd liver membrane protein closely related to the LDL-receptor suggest a physiological role as lipoprotein receptor. EMBO J. 7:4119-4127.

24. Bu, G., Williams, S., Strickland, D.K., and Schwartz, A.L. 1992. Low density lipoprotein receptor-related protein/alpha 2-macroglobulin receptor is an hepatic receptor for tissue-type plasminogen activator. Proc. Natl. Acad. Sci. U. S. A. 89:7427-7431.

25. Hahn-Dantona, E., Ruiz, J.F., Bornstein, P., and Strickland, D.K. 2001. The low density lipoprotein receptor-related protein modulates levels of matrix metalloproteinase 9 (MMP-9) by mediating its cellular catabolism. J. Biol. Chem. 276:15498-15503.

26. Dehouck, B., et al. 1997. A new function for the LDL receptor: transcytosis of LDL across the blood-brain barrier. J. Cell Biol. 138:877-889.

27. Demeule, M., et al. 2002. High transcytosis of melanotransferrin (P97) across the blood-brain barrier. J. Neurochem. 83:924-933.

28. Herz, J., and Bock, H.H. 2002. Lipoprotein receptors in the nervous system. Annu. Rev. Biochem. 71:405-434.

29. Zhuo, M., et al. 2000. Role of tissue plasminogen activator receptor LRP in hippocampal long-term potentiation. J. Neurosci. 20:542-549.

30. Boucher, P., Gotthardt, M., Li, W.P., Anderson, R.G., and Herz, J. 2003. LRP: role in vascular wall integrity and protection from atherosclerosis. Science. 300:329-332.

31. Netzel-Arnett, S., et al. 2002. Collagen dissolution by keratinocytes requires cell surface plasminogen activation and matrix metalloproteinase activity. J. Biol. Chem. 277:45154-45161.

32. Vu, T.H., et al. 1998. MMP-9/gelatinase B is a key regulator of growth plate angiogenesis and apoptosis of hypertrophic chondrocytes. Cell. 93:411-422.

33. Betsuyaku, T., Shipley, J.M., Liu, Z., and Senior, R.M. 1999. Neutrophil emigration in the lungs, peritoneum, and skin does not require gelatinase B. Am. J. Respir. Cell Mol. Biol. 20:1303-1309.

34. Paxinos, G., and Franklin, K.B.J. 2001. The mouse brain in stereotaxic coordinates. Academic Press Inc. San Diego, California, USA. 1-93.

35. Rosenberg, G.A., Estrada, E.Y., Dencoff, J.E., and Stetler-Stevenson, W.G. 1995. Tumor necrosis factor-alpha-induced gelatinase B causes delayed opening of the blood-brain barrier: an expanded therapeutic window. Brain Res. 703:151-155.

36. Nicole, O., et al. 2001. The proteolytic activity of tissue-plasminogen activator enhances NMDA receptor-mediated signaling. Nat. Med. 7:59-64.

37. Matys, T., and Strickland, S. 2003. Tissue plasminogen activator and NMDA receptor cleavage. Nat. Med. 9:371-372.

38. Wong, E.H., et al. 1986. The anticonvulsant MK-801 is a potent $\mathrm{N}$-methylD-aspartate antagonist. Proc. Natl. Acad. Sci. U. S. A. 83:7104-7108.

39. Bu, G. 1998. Receptor-associated protein: a specialized chaperone and antagonist for members of the LDL receptor gene family. Curr. Opin. Lipidol. 9:149-155.

40. Wolburg, H., and Lippoldt, A. 2002. Tight junctions of the blood-brain barrier: development, composition and regulation. Vascul. Pharmacol. 38:323-337.

41. Rubin, L.L., and Staddon, J.M. 1999. The cell biology of the blood-brain barrier. Annu. Rev. Neurosci. 22:11-28.

42. Castellanos, M., et al. 2003. Plasma metalloproteinase-9 concentration predicts hemorrhagic transformation in acute ischemic stroke. Stroke. 34:40-46.

43. Siao, C.J., Fernandez, S.R., and Tsirka, S.E. 2003. Cell type-specific roles for tissue plasminogen activator released by neurons or microglia after excitotoxic injury. J. Neurosci. 23:3224-3242.

44. May, P., Reddy, Y.K., and Herz, J. 2002. Proteolytic processing of low density lipoprotein receptor-related protein mediates regulated release of its intracellular domain. J. Biol. Chem. 277:18736-18743.

45. Coughlin, S.R. 2002. Protease-activated receptors in the cardiovascular system. Cold Spring Harb. Symp. Quant. Biol. 67:197-208.

46. Sobey, C.G., and Cocks, T.M. 1998. Activation of protease-activated receptor-2 (PAR-2) elicits nitric oxide-dependent dilatation of the basilar artery in vivo. Stroke. 29:1439-1444.

47. Jiang, X., et al. 2002. Storage and release of tissue plasminogen activator by sympathetic axons in resistance vessel walls. Microvasc. Res. 64:438-447.

48. Llorente-Cortes, V., Martinez-Gonzalez, J., and Badimon, L. 2000. LDL receptor-related protein mediates uptake of aggregated LDL in human vascular smooth muscle cells. Arterioscler. Thromb. Vasc. Biol. 20:1572-1579. 